\title{
Rotated Upper Central Incisor Complicated by Pathological Migration with Crowding in Upper and Lower Anterior Teeth Treated by Clear Aligners
}

\author{
Cecilia Young* \\ Independent Researcher, Hong Kong
}

*Corresponding author: Cecilia Young, Independent Researcher, Hong Kong. .

Received Date: April 13, 2019

Published Date: May 24, 2019

\begin{abstract}
A Case Report
Pathological tooth migration is difficult to treat; the improvement of the periodontal condition is necessary to stop the disease; fixed appliance gives extra obstacles for oral hygiene practices. Clear aligners make no difference in brushing and flossing during the treatment but have more limitations in tooth movements especially extrusion and derotation. Careful design and flexible treatment strategies for clear aligners are crucial to treat rotated tooth complicated by pathological migrated tooth. This case report showed clear aligners could be used for derotation and extrusion.
\end{abstract}

Keywords: Pathological tooth migration; Extrusion; Clear aligner; Derotation; Orthodontics

\section{Background}

Pathological tooth migration increased between 2.95 to 7.97 times as bone loss increased [1], it is difficult to treat, and the multidisciplinary approach is usually needed [2-4]. The combination of periodontal and orthodontic treatment is being used to improve the periodontal conditions and esthetics [2-6]. Most of the case reports were related to tipping or intrusion of the central incisors [2-6]. Fixed appliances make brushing and flossing more difficult. Oral hygiene practices during clear aligner treatment can be easily maintained. A Meta-analysis concluded that clear aligners were better for periodontal health than fixed appliances [7]. Another systematic review showed a significant improvement of the periodontal health indexes was revealed, in particular when clear aligner treatment was compared to fixed appliances [8], no periodontal adverse effects were observed in the studies using clear aligner treatment [8].

Yunyan et al concluded that clear aligners had advantage in segmented movement of teeth and shortened treatment duration in a systematic review [9], the eight relevant studies used Invisalign [9]. Rossini et al. stated that clear aligner treatment aligns and levels the arches; it is effective in controlling anterior intrusion but not anterior extrusion; it is effective in controlling posterior buccolingual inclination but not anterior buccolingual inclination; it is effective in controlling upper molar bodily movements of about $1.5 \mathrm{~mm}$; and it is not effective in controlling rotation, of rounded teeth in particular [10]. The devices applied in the 11 relevant studies were Invisalign [10].

\section{Case Report}

A 48-year-old lady with mobile rotated and overerupted upper right central incisor visited the dental clinic (Figure 1). She noticed that the tooth became longer and very mobile and wanted to improve the esthetics. She had no complaint with the profile (Figure 2). The upper and lower anterior teeth were crowded (Figure 3).

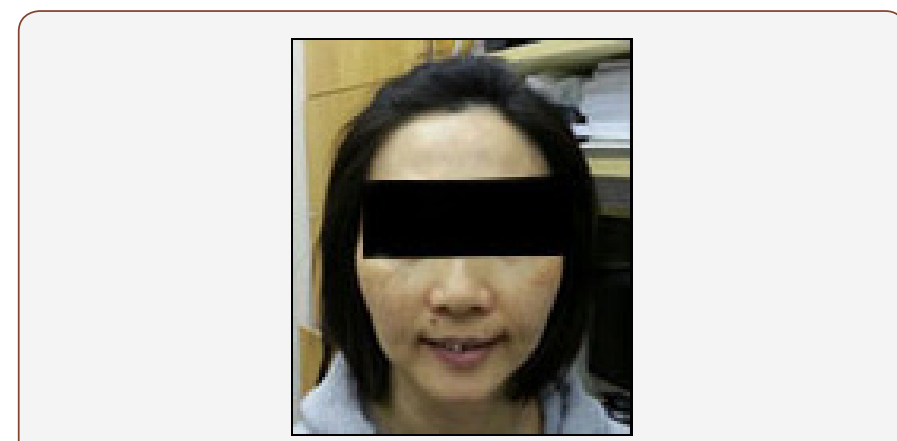

Figure 1: Pre-Op front view. The patient complained about the overerupted 11. 


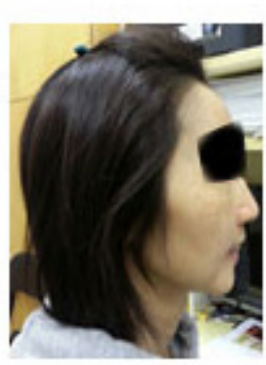

Figure 2: Pre-Op lateral view - The patient had no complaint of the profile.

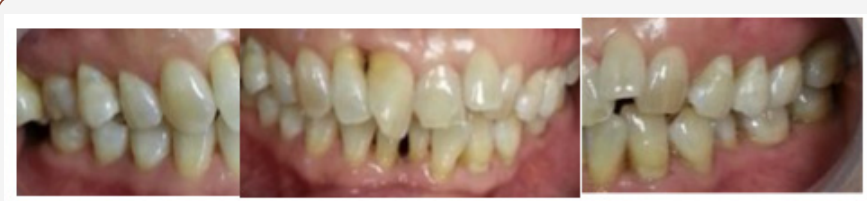

Figure 3: Pre-Op intra-oral views.

\section{Diagnosis}

It was difficult to estimate the original position of the tooth since the upper anterior teeth were crowded, whether it was pathologically migrated or the tooth was originally in an imbalance position that could not be controlled by the lip and finally over erupted and then started the periodontal problem, to help the discussion for the treatment goals, the operator requested the photos in her young age. The 11 was rotated but on the occlusal plane when she was 20 years old (figure 4). Pre-op OPG showed that there was generalised bone loss (figure 5). Pre-op tracing showed slightly retruded Maxilla and Mandible (Figure 6). 11 was rotated (figure 3) and overerupted (figure 3) with mobility.

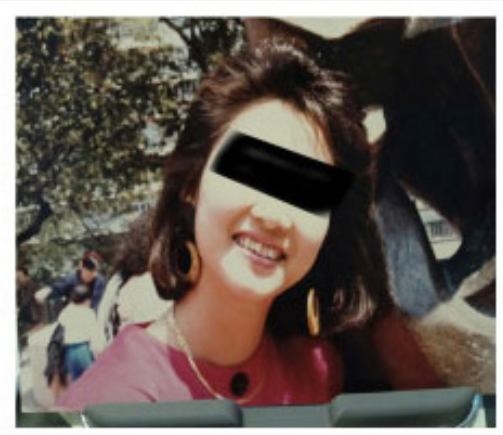

Figure 4: The upper central incisor was rotated but on the occlusal plane.

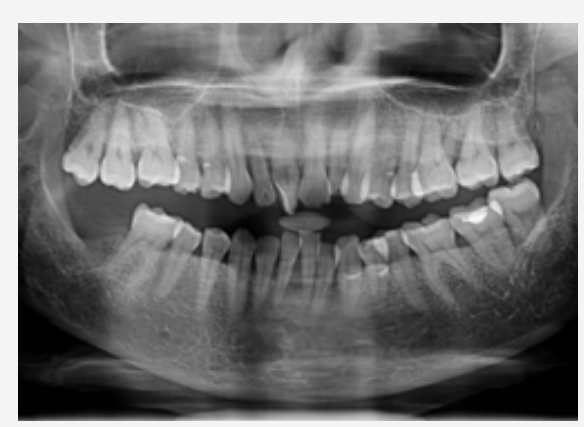

Figure 5: Pre-Op opg showed generalized bone loss.

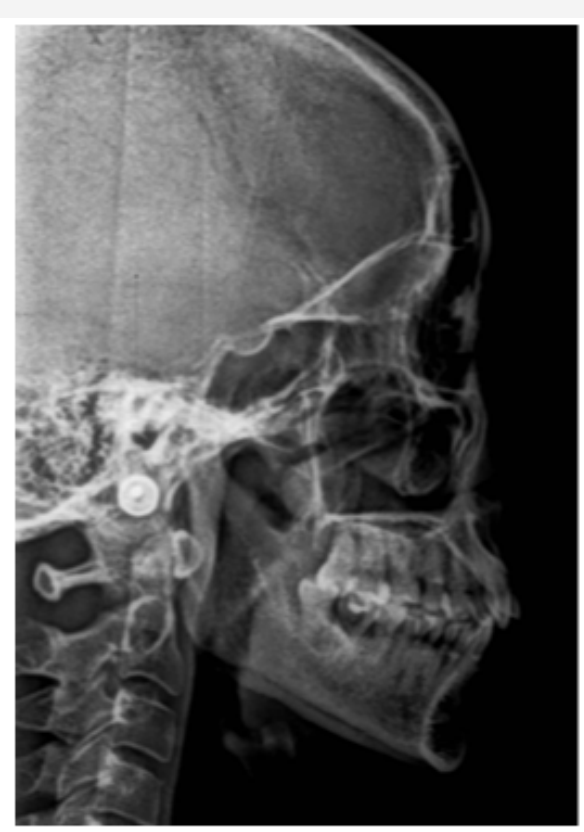

Figure 6: Pre-Op lateral cephalometric.

\section{Treatment Alternatives and Treatment}

Oral hygiene instruction included brushing, flossing and interdental brushing was given, scaling and root planning were performed to improve the periodontal condition. $0.2 \%$ chlorhexidine mouthwash (Corsodyl) twice daily for 5 days was prescribed. Patient was also given with Chlorhexidine 1\% gel (corsodyl gel) $20 \mathrm{ml}$ for adjacent surfaces of 11 and 16, after the finish of the chlorhexidine mouthwash. She requested to improve the esthetics, the poor periodontal condition with attachment loss made it very difficult, a meeting with all her family members included her husband and two daughters were held, pros and cons of different treatment options were discussed. The combination of periodontal with prosthetic treatment most probably needed root canal treatment due to the crowding of upper anterior teeth and the rotated overerupted 11 . Orthodontic treatment will give better alignment. Root canal treatment plus post core and crown will be needed if the unfavorable outcome of pulpal death happens even with light force. Black triangular spaces between teeth were also mentioned. The whole family agreed on the treatment plan of clear aligner (Ecligner) and the need of the retainers. They disagreed with complicated periodontal regenerative procedures and implants for 47.

The pre-op profile was accepted. Orthodontic treatment goals included to align and relieve the upper and lower anterior crowding. The treatment was divided into 2 phases which allowed for fine adjustments, number of steps was increased making sure no excessive force to 11 . The fabrication of the aligners included soft $(0.5 \mathrm{~mm})$, medium $(0.62 \mathrm{~mm})$ and hard $(0.75 \mathrm{~mm})$, each of 1 week per step, non-extraction method but stripping of total $2.5 \mathrm{~mm}$ of the upper teeth was needed for the correction of crowding and alignment. Mild modification during the treatment and slight difference from the expected outcome might happen, the whole family agreed the proposed final set up result. During the 
first treatment phase, the patient felt the hard aligner $(0.75 \mathrm{~mm})$ exerted too much force on 11; then the operator instructed only soft and medium aligners were for used one week and two weeks respectively in each step. The progress was satisfactory. No excessive increase in mobility of the 11 observed. The second set of working impression was taken after the first treatment phase (Figure 7). The treatment progress was satisfactory, but the periodontal condition was not optimal (Figure 7). The patient sent the photo with arrows showed that there was lip biting and requested the operator not to extrude the 21, 22 and 23 in the 21st (Figures 8\&9). Oral hygiene instruction reinforcement, scaling and curettage were performed during the treatment period.

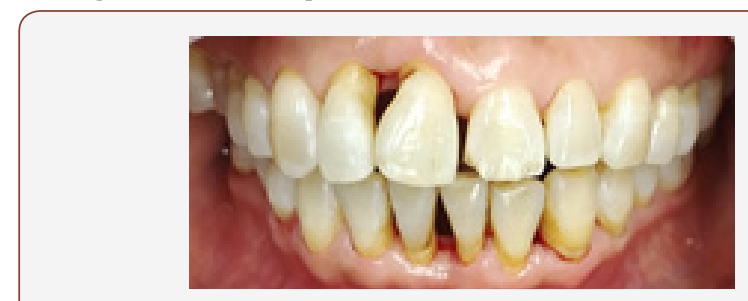

Figure 7: 12-Month Mid-Treatment photo, repeated oral hygiene instruction and scaling improved the periodontal condition but It was not optimal. The second set of working casts were used for the second phase of the teeth set up.
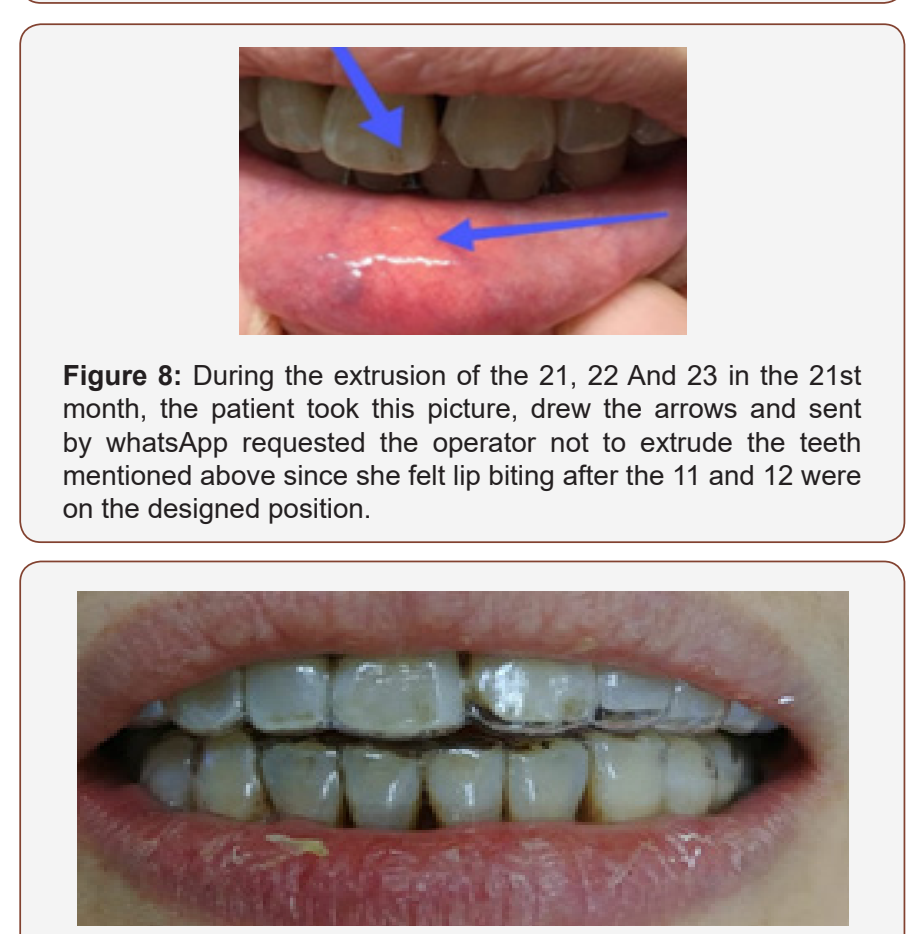

Figure 9: After the explanation, the patient still not psychologically prepared for the small amount further extrusion of 2122 And 23. The operator stopped the extrusion of 21,22 and 23 using attachment and cow catch upon patient's request.

\section{Results}

No adverse outcome observed. The total treatment period was 22 months included the rest for the fabrication of the second phrase aligners. The compliance of wearing the aligners was good; oral hygiene improved a bit but not to optimal. The mobility of 11 decreased from 2 pre-operatively to less than 1 post-operatively. Cold test of all teeth is positive at the review appointment.

\section{Discussion}

Joffe mentioned early experiences of Invisalign $\AA$ included difficulties in treating severely rotated teeth (more than 20 degrees) in 2003 [11]. A systematic review of clear aligners which all the included studies were treated with Invisalign ${ }^{\circledR}$ gave a similar conclusion [10]. However, the differences in design including pressure spots, attachments and the extension of the aligners can combat some of these problems, shown in this case. The extrusion of 12 was satisfactory performing (Figures 8\&9), but the patient refused to further extrude 21, 22 and 23 (Figures 8\&9). The derotation of 11 is successful (Figures 9-15) (Table 1).

Table 1: Pre- and post-treatment cephalometric values.

\begin{tabular}{|c|c|c|c|c|}
\hline & Pre-0p & Post-Op & Ref Value & $\begin{array}{l}\text { Standard } \\
\text { Deviation }\end{array}$ \\
\hline SNA $\left({ }^{\circ}\right)$ & 78.5 & 82.1 & 82 & 2 \\
\hline $\mathrm{SN}-\mathrm{Pg}\left({ }^{\circ}\right)$ & 75.4 & 76.6 & 80 & 5 \\
\hline AN-Pg $\left({ }^{\circ}\right)$ & 3.1 & 5.5 & 2 & 3 \\
\hline $\operatorname{SNA}\left({ }^{\circ}\right)$ & 78.5 & 82.1 & 82 & 2 \\
\hline $\operatorname{SNB}\left({ }^{\circ}\right)$ & 75.2 & 75.8 & 79 & 2 \\
\hline $\operatorname{ANB}\left({ }^{\circ}\right)$ & 3.3 & 6.3 & 3 & 2 \\
\hline Pr-N-A $\left(^{\circ}\right)$ & 3.8 & 2 & 2 & \\
\hline UI-PAL.PLANE $\left({ }^{\circ}\right)$ & 108.2 & 110.2 & 110 & 5 \\
\hline UI-NA mm & 4.3 & 3.1 & 4 & 2 \\
\hline GoGn-Cl $\left({ }^{\circ}\right)$ & 68.6 & 65 & 70 & 4 \\
\hline LI-GoGn $\left({ }^{\circ}\right)$ & 88.6 & 95.6 & 94 & 5 \\
\hline LI-NB (mm) & 4.2 & 7.6 & 4 & 2 \\
\hline Pg-NB (mm) & 0.4 & 1.5 & & \\
\hline SN-GoGn $\left({ }^{\circ}\right)$ & 41.1 & 41.2 & 33 & 4 \\
\hline Pal-Mand Angle $\left({ }^{\circ}\right)$ & 29.3 & 32.1 & 25 & 3 \\
\hline SN-PAL.PLANE $\left({ }^{\circ}\right)$ & 11.7 & 9.1 & 7 & 3 \\
\hline $\begin{array}{c}\text { Mx Base-Occ Pl. } \\
\text { (PP-OP) }\left({ }^{\circ}\right)\end{array}$ & 12.1 & 12 & 10 & 5 \\
\hline $\begin{array}{c}\text { Mand Pl. to Occ } \\
\text { Pl. }\left({ }^{\circ}\right)\end{array}$ & 17.2 & 20.1 & 20 & 5 \\
\hline
\end{tabular}

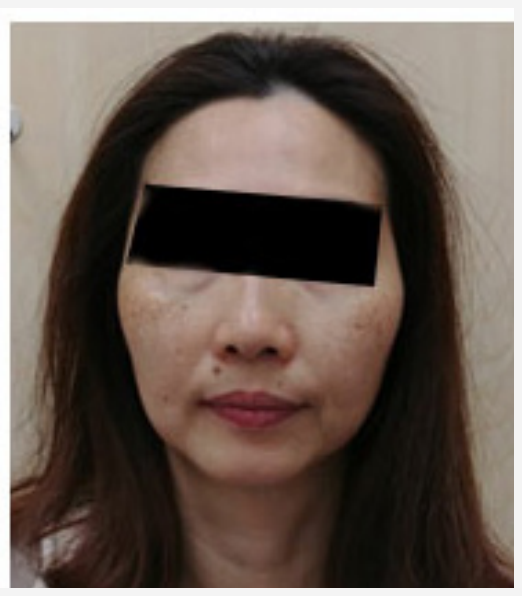

Figure 10: Post-Op frontal view. 


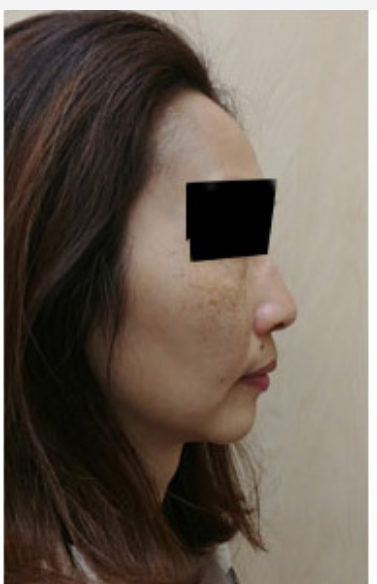

Figure 11: Lateral view Post-Op.

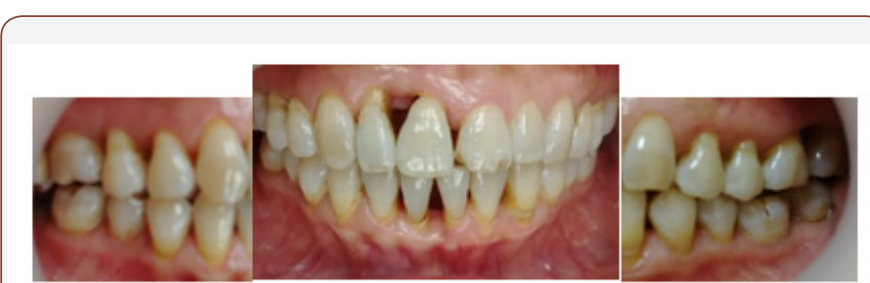

Figure 12: Derotation of 11 was successful. The periodontal condition were still not in optimal despite repeated oral hygiene instruction and scaling. 21, 22 and 23 could be extruded a bit, but the patient did not want it.

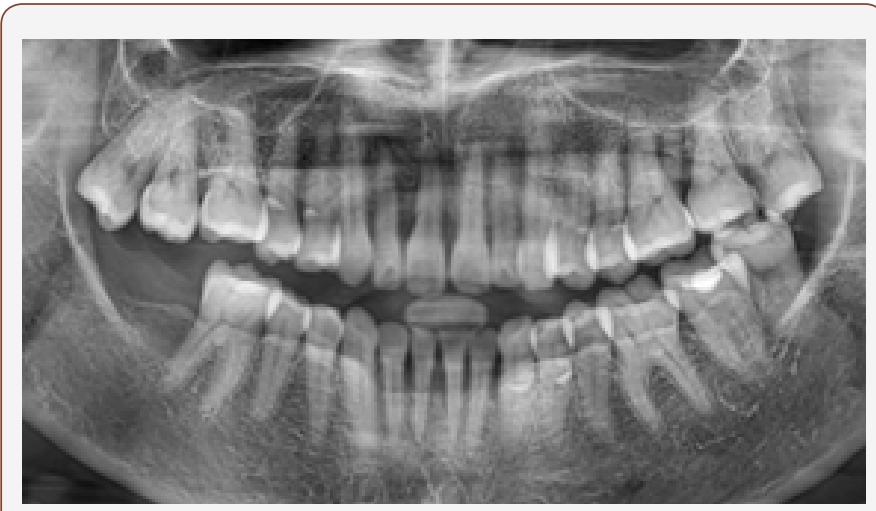

Figure 13: Post-Op OPG, the Patient declined complicated prosthetic and regenerative periodontal treatment and implants, the wearing of clear retainers will maintain the position of 17 and 18.

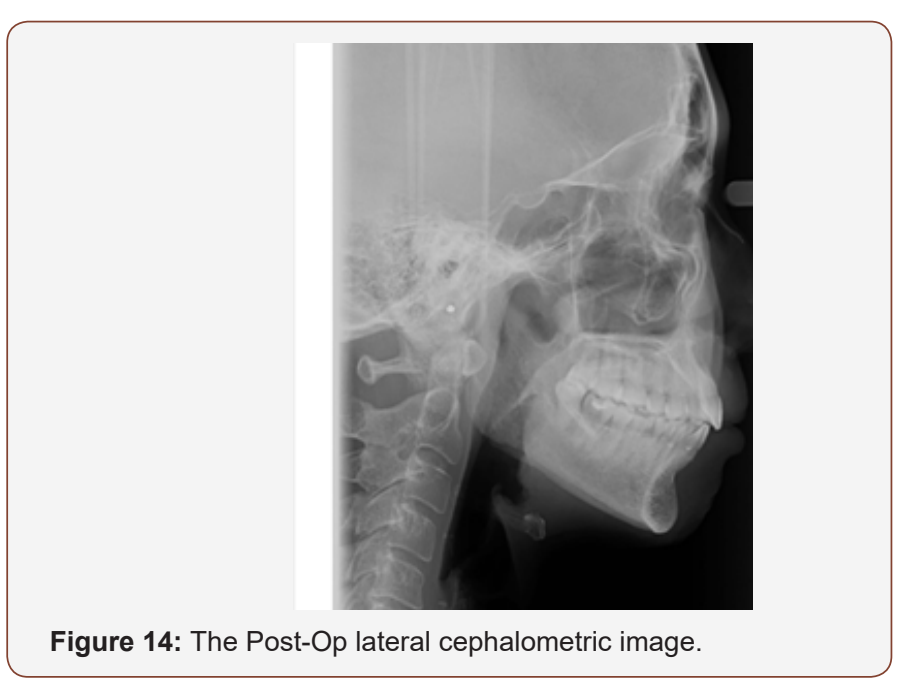

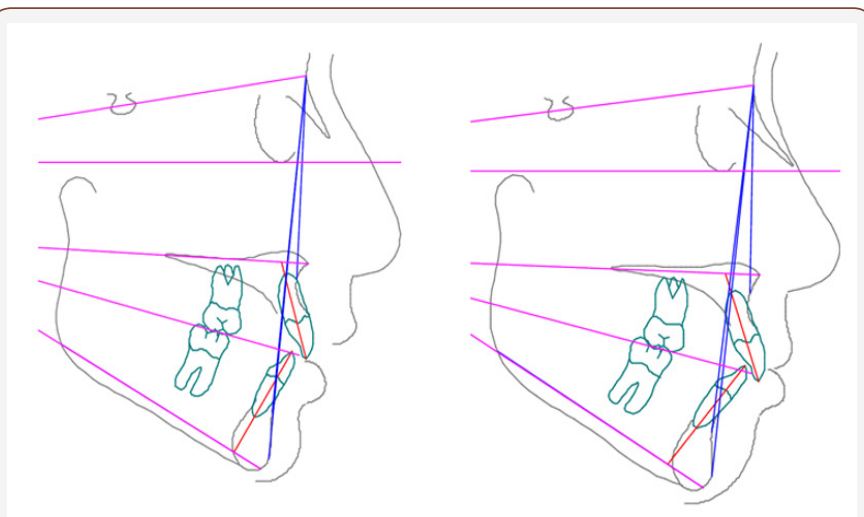

Figure 15: Pre-Op and post-op tracing

The forces delivered by the thick appliances were overall significantly higher than those of the thin materials [12]. The operator instructed the patient not to use the hard aligner (0.75) in each step when the patient complained about the heavy force. Material fatigue does not show a significant difference in the amount of orthodontic tooth movement between the wearing of 1 week or two weeks of the same aligner [13], the wearing of the same aligner for 2 weeks seemed fit for the purpose. Intermaxillary elastics is effective to distalise the teeth combined with clear aligners [14], they were not used to reduce the amount of stripping in the upper arch because it takes longer. The advantage of stripping included improving the gaps between the anterior teeth [15], it is important for the patient.

\section{Conclusion}

With attachments and pressure spot, clear aligners could derotate upper central incisor with almost; extrusion and intrusion are possible. Oral hygiene could be easily maintained during the treatment period. Patient with pathological migration could be managed with clear aligners.

\section{Consent}

Written consent for the use of research, academic and general oral health education purposes was obtained.

\section{Acknowledgement}

None.

\section{Conflict of interest}

None declared. The patient was treated in the author's private dental clinic.

\section{References}

1. Martinez Canut, Carrasquer A, Magán R, Lorca A (1997) A study on factors associated with pathologic tooth migration. J Clin Periodontol 24(7): 492-497.

2. Kim YI, Kim MJ, Choi JI, Park SB (2012) A multidisciplinary approach for the management of pathologic tooth migration in a patient with moderately advanced periodontal disease. Int J Periodontics Restorative Dent 32(2): 225-230.

3. Oh SL (2011) An interdisciplinary treatment to manage pathologic tooth migration: a clinical report. J Prosthet Dent 106(3): 153-158.

4. Jhingta PK, Negi KS, Sharma D, Bhardwaj VK, Vaid S, et al. (2015) Interdisciplinary approach for the treatment of pathologic migration in teeth with advanced periodontal disease. SRM J Res Dent Sci 6: 261-264. 
5. Cao T, Xu L, Shi J, Zhou Y (2015) Combined orthodontic-periodontal treatment in periodontal patients with anteriorly displaced incisors. Am J Orthod Dentofacial Orthop 148(5): 805-813.

6. Lee JW, Lee SJ, Lee CK, Kim BO (2011) Orthodontic treatment for maxillary anterior pathologic tooth migration by periodontitis using clear aligner. J Periodontal Implant Sci 41(1): 44-50.

7. Jiang Q, Li J, Mei L, Du J, Levrini L (2018) Periodontal health during orthodontic treatment with clear aligners and fixed appliances: A metaanalysis. J Am Dent Assoc 149(8): 712-720.

8. Rossini G, Parrini S, Castroflorio T, Deregibus A, Debernardi CL (2015) Periodontal health during clear aligners treatment: a systematic review. Eur J Orthod 37(5): 539-543.

9. Ke Y, Zhu Y, Zhu M (2019) A comparison of treatment effectiveness between clear aligner and fixed appliance therapies. BMC Oral Health 19(1): 24 .
10. Rossini G, Parrini S, Castroflorio T, Deregibus A, Debernardi CL (2015) Efficacy of clear aligners in controlling orthodontic tooth movement: a systematic review. Angle Orthod 85(5): 881-889.

11. Joffe L (2003) Invisalign: early experiences. J Orthod 30(4): 348-352.

12. Hahn W, Dathe H, Fialka Fricke J, Fricke Zech S, Zapf A (2009) Influence of thermoplastic appliance thickness on the magnitude of force delivered to a maxillary central incisor during tipping. Am J Orthod Dentofacial Orthop 136(1): 12.e1-7.

13. Drake CT, Mc Gorray SP, Dolce C, Nair M, Wheeler TT (2012) Orthodontic tooth movement with clear aligners. ISRN Dent 2012: 657973.

14. Luca Lombardo, Anna Colonna, Antonella Carlucci, Teresa Oliverio (2018) Class II subdivision correction with clear aligners using intermaxilary elastics. Prog Orthod 19(1): 32.

15. Georgiadis AA, Darmanin P, Topouzelis N, Ioannidou Marathiotou I (2015) Indication and Technical Application of Stripping. Balk J Dent Med 19: 3-7 